(EMS) transport. Methods: A retrospective cohort study with suspected STEMI patients monitored with prehospital serial ECGs was performed from August 2006 to December 2013. The data was extracted from UCCSPU clinical databases and verified by an emergency physician supervisor. During EMS transport, the serial ECG monitoring system automatically produced and transmitted every 2 minutes a 12-lead ECG without artefacts. STEMI criteria were based on the Third Universal Definition of Myocardial Infarction. Dynamic ECG change was defined as an ST-segment elevation or depression that meets diagnostic criteria (eg. initial non STEMI (NSTEMI) changing to STEMI and vice versa). Results: Among the 752 patients identified with suspected STEMI, 728 $(96.8 \%)$ were included in the study due to missing data. The majority $(614 / 728 ; 84.3 \%)$ had a consistent ST segment without significant dynamic changes throughout transport, of which 521 were identified as STEMI and 93 as NSTEMI. The remaining 114 patients (15.7\%) had dynamic ECG changes: 41 (36\%) evolved from NSTEMI to STEMI, 40 (35.1\%) changed from STEMI to NSTEMI, and 33 (28.9\%) had more than one dynamic ST-segment change. Overall, 59 patients $(8.1 \%)$ had a final STEMI ECG diagnosis after an initial NSTEMI ECG interpretation. Conclusion: In this study, the serial ECG system enabled the remote diagnosis of STEMI in $8.1 \%$ of patients during EMS transport following an initial NSTEMI diagnosis. Serial monitoring of dynamic changes can allow for more rapid diversion to primary percutaneous coronary intervention facilities, potentially improving patient outcomes. Further studies are needed to evaluate the clinical impact, and costs and benefits of implementing this technology.

Keywords: ECG interpretation, STEMI, emergency medical services (EMS)

\section{MP029 \\ Mobilizing citizens, decision-makers and healthcare professionals to find solutions for improving emergency care in a remote Northern emergency department: a pilot study \\ R. Fleet, MD, PhD, C. Turgeon-Pelchat, F.K. Tounkara, J. Trottier, J. Ouellet; Université Laval and CHAU Hôtel Lieu de Lévis, Lévis, QC}

Introduction: Our national study of rural EDs showed they have limited access to CT scans, ICU, and most specialities, while Level 1 and Level 2 trauma centers are on average $300 \mathrm{~km}$ away. However, equivalent information on Northern/remote EDs is scarce. Objectives: This pilot study aimed to: 1) describe local access to services; and 2) mobilize citizens, decision-makers and healthcare professionals to envisage solutions to improving emergency care in a Northern/remote hospital. Methods: This descriptive, qualitative study was performed in a northern ED in Quebec. The head nurse received a prevalidated questionnaire about access to specific services, ED and patient characteristics (Objective 1). Focus groups (5) and individual interviews (11) were conducted with citizens/patients, decision-makers, physicians, nurses, paramedics, pharmacists, and mental health workers (Objective 2). Descriptive statistics are reported as means, medians and percentages for Objective 1. A thematic analysis was conducted for Objective 2. Results: Objective 1: The city (population 2875) is a mining community $962 \mathrm{~km}$ from Quebec City. The 2010 annual ED census was 6692. Proportions of patient visits at triage levels $1-5$ were $0.2 \%, 3.2 \%, 13.4 \%, 25.4 \%$ and $56.7 \%$ respectively. The ED was staffed by one physician and two nurses per shift. The hospital had 24/7 access to basic X-ray and laboratory but no local access to speciality care, ICU, CT scan or ultrasound, with nearest services $28 \mathrm{~km}$ away. Objective 2: Analysis of qualitative data highlighted concerns for personal safety; telecommunication problems; lengthy transports; limited access to in-service training, advanced imaging, and consultants; and recruitment and retention difficulties.
Solutions included pre-hospital training, telemedicine, protocols, and networking with academic centers/medical schools, North Shore colleagues, and Labrador City Hospital. Conclusion: This isolated northern ED has limited access to services. Valuable qualitative information obtained enabled us to better understand the challenges and explore solutions towards improving Northern/remote emergency care.

Keywords: Northern emergency department, improving emergency care, mobilization

\section{MP030}

Problems in paramedic-physician telecommunication

D. Eby, MD, PhD, J. Robson, M. Columbus, PhD; Western University, London, ON

Introduction: Clear paramedic-physician telecommunications (patches) are critical in systems utilizing on-line medical control. In systems using extensive medical directives individual paramedics patch infrequently. Investigations of specific problem calls indicated that communication problems were more common than believed. Existing literature on this topic is sparse. This project is a quality assurance exercise undertaken to understand the extent and nature of problems in paramedic-physician telecommunications Methods: Retrospective analysis of anonymized transcriptions made from MP3 audio files recorded as part of normal operating procedures by the Central Ambulance Communication Centre during January-March 2014. All calls where telecommunication occurred between paramedics from 4 ambulance services and base hospital physicians providing on-line medical oversight during ambulance calls were included. Transcripts were read multiple times and data extracted onto spreadsheets for frequency analysis. Further thematic framework analysis of emergent themes was done. Results: All 42 patches were transcribed and used for analysis. 36 (85.7\%) were for termination of resuscitation orders, $4(9.5 \%)$ were for advice, and $2(4.8 \%)$ were for orders not covered by medical directives. Communication problems were identified in $40(95.2 \%)$ patches. Most had multiple problems. These included disconnections (23.8\%), difficulty hearing one another (40.5\%) - indicated by phrases such as "sorry?" "what?", "I can't hear you" - or caused by individuals interrupting each other (83.3\%), and talking simultaneously (47.6\%). Signaling the end of "talk turns" - such as "10-4" or "over" - was never used. Instead, terms like "yah" and "OK" were used. When communication went awry, time was spent trying to repair the mis/poor communication. This led to repeating information or attempting to 'sell' the case by providing information unnecessary for decision making such as during a request for termination of resuscitation, "there is vomit on the floor". Conclusion: Paramedic-physician telecommunication problems were extremely common. They involved technical (mechanical problems) and human factors (disorganized radio 'technique'). The high incidence of telecommunication problems identified is concerning. Critical clinical decisions (e.g. ceasing resuscitation) depend on clear communication. Further study of these issues is warranted.

Keywords: telecommunication, paramedic, patch

\section{MP031}

Synovial fluid analysis in the diagnosis of septic arthritis: comparing local data to the literature

E. Logan, MD, J. Fedwick, MD, PhD; University of Calgary, Calgary, AB

Introduction: A hot, painful, swollen joint is a common presentation to the emergency department. Of the potential etiologies, septic arthritis (SA) is the most devastating. Prompt diagnosis and treatment are 
essential to improve outcomes. Both culture proven and clinically suspected SA are thought to have the same prognosis, with similar morbidity and mortality estimates. No clinical exam or serum lab finding has the sensitivity or specificity to diagnose or exclude SA. Instead, diagnosis relies mainly on joint aspiration and synovial fluid analysis. A synovial white blood cell count (sWBC) greater than 50,000 cells/ microliter is suggestive of SA and organisms seen on gram stain or growing in culture effectively makes the diagnosis. However, culture and gram stain are positive in only $67 \%$ and $50 \%$ of cases respectively. The objective of this study was to analyze the accuracy of synovial fluid analysis in our local practice environment. Methods: All those encounters with diagnoses related to SA at four adult emergency departments in Calgary between 2013-2014 were reviewed. Hospital records were analyzed for synovial analysis, antibiotic usage and surgical procedures. Results: Of 286 encounters, 87 were determined to satisfy the definition for SA in that culture was positive, gram stain was positive or clinical findings lead to treatment with antibiotics and/or surgical intervention. Gram stain was positive in $22 \%$ of cases with cultures positive in $51 \%$ of patients. sWBC were less than 50000 in $55 \%$ of cases and less than 25000 in $24 \%$ of cases. Of 88 gram stains performed, $28 \%$ were negative but had positive culture. All positive gram stains were associated with positive cultures. Conclusion: Culture, gram stain and sWBC of patients diagnosed with SA in Calgary show differences compared with the published literature. In Calgary, the majority of SA diagnoses were made clinically. The sWBC is central to making the diagnosis. Interestingly, $55 \%$ of patients diagnosed with SA had a count less than 50,000 . It remains unclear what features of history, physical exam, imaging and lab analysis lead to the diagnosis of SA in these cases. Future studies will focus on these outliers to see if a more appropriate diagnostic algorithm would be useful in Calgary. Collaboration between infectious disease specialists, orthopedics, and emergency departments guided by local data is needed to ensure accurate and timely diagnosis.

Keywords: septic arthritis, diagnosis

\section{MP032}

Do urine cultures in the emergency department change management of young women with symptoms of uncomplicated urinary tract infection?

E. Poon, MD, L. Self, MD, S.L. McLeod, MSc, S. Caine, MD, B. Borgundvaag, $\mathrm{PhD}, \mathrm{MD}$; University of Toronto, Toronto, $\mathrm{ON}$

Introduction: Current guidelines do not recommend the routine use of urinary cultures in the management of uncomplicated urinary tract infection (UTI) in premenopausal, non-pregnant women. Complicating factors include atypical presentation, structural abnormalities or recent recurrent infection/antibiotic use. The objective of this study was to determine the number of urine cultures ordered for women who presented to the emergency department (ED) with symptoms of uncomplicated UTI, and whether a culture result impacted subsequent management. Methods: This was a retrospective chart review of women aged 18-39 presenting to one of two academic EDs with a discharge diagnosis of uncomplicated UTI from Jan-Dec 2014. Patients were excluded if any of the following were documented: pregnancy, fever, immunocompromised state, diabetes mellitus, absence of lower urinary tract symptoms, ED administration of intravenous antibiotics, a previous UTI treated with antibiotics in the last 90 days, two weeks post-partum or post-instrumentation. Results: Of the 512 charts included in the analysis, $494(96.5 \%)$ patients had a urinalysis, of which $463(93.7 \%)$ had positive leukocyte esterase and $90(18.2 \%)$ had positive nitrites. 370 patients $(72.3 \%)$ had urine cultures performed, of which $236(63.8 \%)$ were positive. 505 (98.6\%) patients received antibiotics $(53.9 \%$ Macrobid; $22.6 \%$ Ciprofloxacin; $15.0 \%$ Septra; $6.7 \%$ other; $1.8 \%$ not documented). $7(1.9 \%)$ cultures grew organisms resistant to the prescribed antibiotic; $2(0.5 \%)$ patients received new prescriptions. Conclusion: For the majority of young female patients with uncomplicated UTI, urine cultures did not change management. Almost all of these patients had a positive leukocyte esterase and were treated with antibiotics, yet approximately $40 \%$ of the patients tested did not return positive urine cultures, suggesting that better algorithms for the diagnosis of UTI in the ED are required. Unnecessary treatment with antibiotics is expensive, contributes to the development of multidrug resistant organisms, and exposes the patient to the unnecessary risks of possible allergic reactions, drug interactions and side effects.

Keywords: urinary tract infection, culture, antibiotic

\section{MP033}

The use of femoral nerve blocks in the emergency department for hip fracture patients

A. Sandre, BSc, C. Thompson, MSc, S.L. McLeod, MSc, B. Borgundvaag, MD, PhD; Schwartz/Reisman Emergency Medicine Institute, Toronto, ON

Introduction: Hip fractures affect over 30,000 Canadians each year. Delirium, or acute confusion, occurs in up to $62 \%$ of patients following a hip fracture. Delirium substantially increases hospital length of stay and doubles the risk of nursing home admissions and death. Previous studies have shown that regional anesthesia is the optimal pain management strategy for hip fracture patients and has been shown to independently reduce the rate, severity and duration of delirium. However, very few emergency physicians (EPs) have the necessary training and experience to use regional anesthesia for hip fracture in the emergency department (ED). The objective of this study was to determine the number of femoral nerve blocks performed within the ED for the management of hip fracture patients. Methods: This was a retrospective chart review of patients aged 65 years and older, presenting to an academic ED (annual census 60,000) with a discharge diagnosis of hip fracture from January $1^{\text {st }} 2014$ to July $31^{\text {st }} 2015$. Results: Of the 243 hip fractures included in this study, mean (SD) age was 82.9 (8.2) years and $187(77.0 \%)$ were female. The majority $(214,88.1 \%)$ of patients arrived to the ED by ambulance and $182(74.9 \%)$ were categorized as CTAS 3. The most common analgesics used in the ED were intravenous (IV) hydromorphone $(51.4 \%)$, IV morphine $(32.1 \%)$, or dual therapy with both IV hydromorphone and IV morphine (4.9\%). Femoral nerve blocks were initiated for $13(5.3 \%)$ patients and successfully completed in $12(4.9 \%)$ patients in the ED. Median (IQR) ED and hospital length of stay was $5.0(3.7,6.6)$ hours and $6.0(4.1,10.2)$ days, respectively. Forty-three $(17.7 \%)$ patients experienced in-hospital acute delirium. Conclusion: Despite evidence to suggest regional anesthesia may be the optimal pain management strategy for hip fracture patients, the use of femoral nerve blocks in the ED remains low. Future research should attempt to elucidate barriers to use of this procedure by emergency physicians.

Keywords: analgesia, hip fracture, delirium

\section{MP034}

What is the diagnostic accuracy of Canadian emergency physicians and cardiologists interpreting potential acute ST-elevation myocardial infarction (STEMI) electrocardiograms? 